www.jmscr.igmpublication.org

Impact Factor 5.244

Index Copernicus Value: 83.27

ISSN (e)-2347-176x ISSN (p) 2455-0450

crossref DOI: http://dx.doi.org/10.18535/jmscr/v4i8.65

\title{
Radiographic Findings of Peri-Apical Lesions and Its Correlation to Clinical Findings
}

\author{
Authors \\ Lakshmi Prabha. $\mathbf{J}^{1^{*}}$, Dr. S.Karpagavalli ${ }^{2}$ \\ ${ }^{1} 3^{\text {rd }}$ Yr Bds, Saveetha Dental College, Poonamalle, Chennai-77 \\ ${ }^{2}$ Department Of Oral Medicine And Radiology, Saveetha Dental College, Poonamalle, Chennai-77 \\ Corresponding Author \\ Lakshmi Prabha.J \\ Saveetha Dental College \\ Email: prabha_jeeva@rediffmail.com,Ph no: 7708147895
}

\begin{abstract}
Aim: The aim of this study is to correlate the radiological and clinical findings of various periapical lesions of the oral cavity.

Objective: The objective of the study is to analyse the various radiographic and clinical interpretation and correlate the findings by using radiographs. Around 30 radiographs of patients with periapical lesions was selected irrespective of age, sex and race and was evaluated to find the radiographic and to correlate the radiographic findings with its clinical findings.

Materials and method: 30 patients above the age of 18years who were diagnosed with periapical lesions were selected. Clinical and radiographic photographs of these patients were collected and compared.

Results: Among the 30 patients, prevalence of periapical abscess was predominant (80\%). Radio graphically significant finding was the presence of PDL widening observed in $86 \%$ of the cases. Periodontal ligament space was seen in 3\% of the cases. Loss of lamina dura was seen in 37\% of the cases whereas pulp calcification was observed in 3\% of the cases. Clinically, all the lesions were red in colour with regular margins with 27(90\%) patients having tenderness on palpation. Around 26(87\%) lesions were localised and 23(77\%) patients had sinus discharge.

Conclusion: The periapical lesions found in the oral cavity are seen to occur predominantly in patients with poor periodontal status irrespective of age, sex and race. It is important to study the progress of the sequelae and provide accurate diagnosis for better prognosis. Hence this study is done to emphasis the importance of correlation between radiographic findings and clinical findings.
\end{abstract}

\section{Introduction}

Pulpo-periapical pathologies are inflammatory conditions caused due to necrotic dental pulp which spreads to the periapical region of the tooth. [1] The cause for periapical pathology may be variable including idiopathic, immunological as well as reaction to certain foreign substances. ${ }^{(2)}$ Various diseases seen in the periapical region are periapical abscess, periapical cyst, periapical granuloma and periapical periodontitis. Of these, Periapical cyst and granuloma are lesions most commonly found at the apices of non-vital 
teeth.These may be observed in the radiograph as abnormal radiolucencies. [1][3]) A careful evaluation of the disease is required for correct diagnosis and will further help in planning its treatment. In order to obtain a definitive diagnosis, a dentist must be aware of normal radiograph and all abnormalities of the periapical region. [4][5] Hence this study is done to compare the clinical and radiographic findings of these periapical lesions.

\section{Materials and Method}

Patientswith periapical pathology who visited a private dental college in chennai were selected for the study. Around 30 patients above the age of 18 years who were diagnosed with periapical pathology were selected. Intra oral periapical radiographs taken using bisecting angle technique and clinical photographs of these patients were collected and compared to obtain the results. ${ }^{[4][3]}$

\section{Results}

Of the 30 patients, $80 \%$ of the lesions were periapical abscess, $13 \%$ were periapical periodontitis, 3\% were periapical cyst and 3\% were periapical granuloma. (Fig.1) Radio graphically, periodontal widening was seen in $86 \%$ whereas presence of periodontal space was seen in $3 \%$ of the cases. Loss of lamina dura was seen in $37 \%$ of the cases. Pulp calcification was seen in $3 \%$ of the cases. (fig. 2 )

Clinically, all the lesions were red in colour with regular margins. About 27(90\%) patients had tenderness while $3(10 \%)$ patients were asymptomatic. Around 26(87\%) lesions were localised of which $4(13 \%)$ lesions were circular, $16(53 \%)$ were ovoid and 10(33\%) were irregular. $23(77 \%)$ patients had sinus opening of which $15(50 \%)$ patients had pus discharge and $8(27 \%)$ patients had serous discharge. (Fig.3)

Figure1: Incidence of periapical lesions in 30 patients

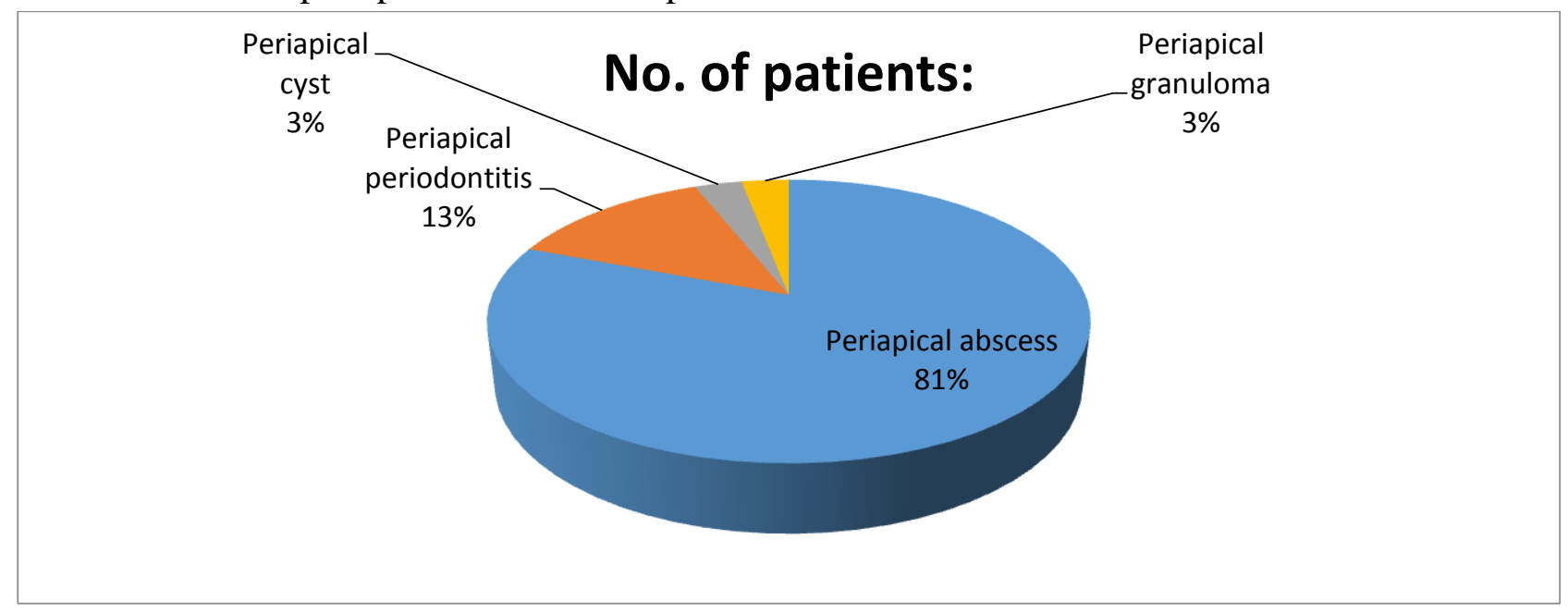

Figure2: radiographic findings of the lesions in the selected patients

Title

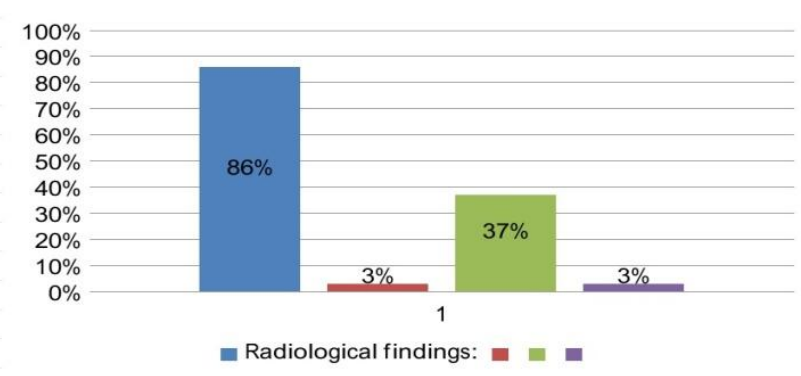


Figure3: clinically findings of the lesions in the selected patients

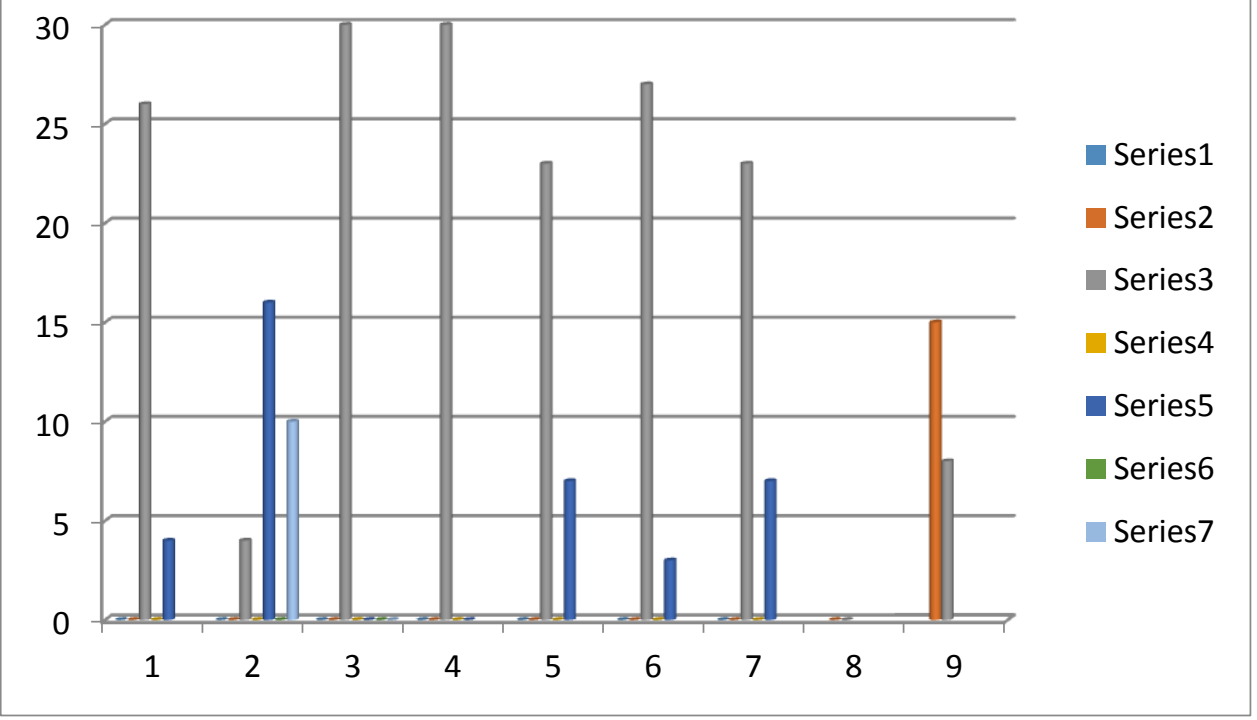

\section{Discussion}

Radiographic interpretation of a clinical pathology can be mastered by a systemic and careful approach. ${ }^{[6]}$

A definitive diagnosis can be achieved only if a proper radio-diagnosis is done. The present study was carried out to compare the clinical and radiographic diagnostic features of periapical lesions to obtain correct diagnosis for efficient treatment planning. ${ }^{[7]}$

Of the 26 cases which had Periodontal ligament widening and localised spread, 24 were identified as periapical abscess with 23 cases reporting of pus discharge. Tenderness was seen in 27 cases whereas 3 patients were asymptomatic. This indicates that any disturbance of the periodontal ligament causes tenderness irrespective of the type of lesion. All 30 patients had regular margins and redness of the swelling irrespective of periodontal ligament widening or space thickening. It was observed thatLoss of lamina dura also did not affect the colour and margins of the swelling. ${ }^{[2][6]}$

\section{Conclusion}

In order to avoid mistreatment, dentists should be aware of all the features of lesions of the periapical regions. ${ }^{[7][8]}$ A good knowledge of anatomical considerations and pathological sequel of periapical lesions coupled with a trained systemic approach to radiographic interpretation will aid in diagnosis and treatment planning. ${ }^{[8]}$ Hence this study was done to correlate clinical and radiographic findings and to emphasise the significance of these in features in the diagnosis of these periapical lesions.

\section{References}

1. Edmund Peters, Monica Lau; Histopathologic Examination to confirm diagnosis of Periapical lesions: A review; J Can Dent Assoc 2003, 69 (9): 598-600.

2. Raphel Carlos Comelli LIA, Juliana Maira Q. GARCIA, Manoel D. SOUSA-NETO, Paulo Cesar SAQUY, Renata Hebling MARINS, Wagner grandinni ZUCOLLOTTO; Clinical and Radiographic and Histological Evaluation of Chronic Periapical Inflammatory Lesions; J Appl Oral Sci 2004, 12 (2) 117-20.

3. Nausheen Mobeen; Incidence of common Periapical lesions- An intraoral periapical study; J Pharm Sci and Research Vol. 7 (8) 2015.

4. Seyed Mohammed Razani, Sima Kiani, Saeedeh Khalesi; Periapical lesion: A review of Clinical, Radiographic and Histological Features; Dent Res. 2015 June; 7 (1) ajdr 19435. 
5. S. Jagadhish, H. Mural, J. Karthik; Resolution of Periapical Pathology- A non-surgical approach.

6. Hans-Goran, Grondahl, Sisko Huumonen; Radiographic manifestations of Periapical Inflammatory Lesions; Entodontic topics 2004 1601-1538.

7. AO Akinyamaju, SO Gbadebo, BF Adeyemi; Periapical lesions of the Jaws, A review of 104 cases in IBADAN; Ann Ib Post grad Med. 2014 Dec; 12 (2). 115-119.

8. Subashini Suyambukesan, Gopu Chandran, LeninPerumal, Elangovan Somasundaram, Nagedran, Jayavel Pandian, $\mathrm{T}$ Manigandan; Analysing Periapical Lesions on Intra oral periapical radiographs: Incongruity in Diagnosis; 10.5005/ jp journals-10011-1330. 